Revue d'archéologie préhistorique

\title{
Un nouveau faciès lamellaire du début du Paléolithique supérieur dans les Balkans
}

A new bladelet facies of the Early Upper Palaeolithic from the Balkans

Nikolay Sirakov, Tsenka Tsanova, Svoboda Sirakova, Stanimira Taneva, Ivaylo Krumov, Irena Dimitrova et Natalia Kovatcheva

\section{OpenEdition}

\section{Journals}

Édition électronique

URL : https://journals.openedition.org/paleo/565

DOI : $10.4000 /$ paleo.565

ISSN : $2101-0420$

Éditeur

SAMRA

Édition imprimée

Date de publication : 30 décembre 2007

Pagination : 131-144

ISSN : 1145-3370

Référence électronique

Nikolay Sirakov, Tsenka Tsanova, Svoboda Sirakova, Stanimira Taneva, Ivaylo Krumov, Irena Dimitrova et Natalia Kovatcheva, « Un nouveau faciès lamellaire du début du Paléolithique supérieur dans les Balkans », PALEO [En ligne], 19 | 2007, mis en ligne le 23 avril 2009, consulté le 26 juin 2021. URL : http://journals.openedition.org/paleo/565 ; DOl : https://doi.org/10.4000/paleo.565

\section{(c)}

PALEO est mis à disposition selon les termes de la licence Creative Commons Attribution - Pas d'Utilisation Commerciale - Pas de Modification 4.0 International. 


\title{
UN NOUVEAU FACIÈS LAMELLAIRE DU DÉBUT DU PALÉOLITHIQUE SUPÉRIEUR DANS LES BALKANS
}

\author{
Nikolay SIRAKOV ${ }^{(1)}$, Tsenka TSANOVA ${ }^{(2)}$ et Svoboda SIRAKOVA ${ }^{(1)}$, \\ Stanimira TANEVA ${ }^{(1)}$, Ivaylo KRUMOV(1), \\ Irena DIMITROVA ${ }^{(1)}$, Natalia KOVATCHEVA ${ }^{(1)}$
}

\begin{abstract}
Résumé : Après Bacho Kiro et Temnata, la grotte de Kozarnika est la troisième grande séquence pléistocène récemment établie dans la région de l'est des Balkans. La fouille, menée dans le cadre d'une coopération entre l'Académie bulgare des sciences - Institut d'Archéologie à Sofia et UMR 5199 du CNRS, PACEA - Université Bordeaux 1, a permis la découverte d'une séquence, datant du Paléolithique inférieur (non Acheuléen), Paléolithique moyen (Moustérien - Levallois) et du Paléolithique supérieur, daté entre 39 et 11 Ka BP (Guadelli et al. 2005). Le niveau VII, localisé à la base du Paléolithique supérieur, daté entre 39 et $36 \mathrm{Ka}$ BP a livré une industrie lithique à débitage et outillage lamellaire, inédite à l'échelle régionale. Cet outillage est composé de pièces à dos et à retouche fine, abrupte, et semi-abrupte, parfois bilatérale directe appointant ou alterne. Dénommée "Kozamikien " en attente d'études plus complètes, cette industrie présente des analogies dans la composante lamellaire tant avec l'Ahmarien ancien du Levant qu'avec certains ensembles de proto-Aurignacien au sud de l'Europe. En l'absence d'autres éléments caractéristiques de l'Aurignacien typique, cette industrie montre des tendances technologiques et typologiques dont on trouve la continuité dans les niveaux sus-jacents gravettiens de la séquence. Le Kozarnikien peut donc représenter une étape précoce de formation de technocomplexe lamellaire à pièces à dos. Le Kozarnikien suggère aussi qu'on ne peut plus soutenir le rôle exclusif de l'Aurignacien dans le passage du moustérien européen au Paléolithique supérieur. II devient de plus en plus évident qu'au tout début de cette transformation, ont été engagées des traditions culturelles différentes et précédant l'Aurignacien.
\end{abstract}

Mots-clés : Balkans, Kozarnika, Paléolithique supérieur initial, Gravettien, production lamellaire.

Abstract: A new bladelet facies of the Early Upper Palaeolithic from the Balkans. With Bacho Kiro and Temnata, Kozranika cave is the third major pleistocene sequence recently investigated in the Eastern Balkans. Excavations, conducted in collaboration of the Bulgarian Academy of sciences-National Institute of Archaeology at Sofia and the University of Bordeaux 1- Institute of Prehistory and Quatemary Geology -CNRS, PACEA, UMR 5199, revealed a sequence starting with non-Acheulean Lower Palaeolithic (LP), Levaloisian Moustérian Middle Palaeolithic (MP) and Upper Palaeolithic(UP) dated between $39 \mathrm{Ky} \mathrm{BP}$ and $11 \mathrm{Ky}$ BP (Guadelli et al. 2005). Level VII, at the base of the UP (39-36 Ky BP) contains an assemblage locally unknown, characterized by a bladelet technology and tools made on bladelets: regular backed pieces and bladelets with thin, abrupt or semi-abrupt, often bilateral convergent or alternate retouch. Named temporarily "Kozamikian" awaiting for further studies, this assemblage shows some analogies with the archaic Ahmarian from Levant and SouthEuropean Proto-Aurignacian as well. Given the lack of diagnostic artefacts of typical Aurignacian, this assemblage shows technological and typological tendencies observed in the overlaying Gravettian industries. The Kozarnikian might be considered as a possible precursor of technocomplexes with bladelet technology and backed pieces and thus,suggesting that the role of Aurignacian in transition between the European Mousterian and the UP is not unique nor exclusive. It becomes more and more obvious that, at the beginning of this transformation, preceding the Aurignacian, different cultural traditions where involved.

Key-words: Balkans, Kozarnika, Initial Upper Paleolithic, Gravettian, microblade production.

\section{INTRODUCTION}

Dans le débat actuel qui agite la communauté scientifique à propos de l'émergence du Paléolithique supérieur, la production systématique de lamelles est un aspect important. Elle est souvent considérée comme liée à l'évolution des stratégies de l'exploitation du milieu par extension des industries à composante lamellaire qui sont habituellement attribuées à l'homme anatomiquement moderne en Europe.
Caractériser les plus anciennes productions lamellaires du début du Paléolithique supérieur est donc essentiel pour la compréhension des modalités techniques du passage technologique du Paléolithique moyen au Paléolithique supérieur, mais aussi pour discuter les modèles du peuplement de l'Europe par l'homme anatomiquement moderne.

Le territoire des Balkans, situé au carrefour de l'Europe, du Proche-Orient et de l'Eurasie steppique a, dans ce cadre, une position importante.

(1) Institut National et Musée d'Archéologie à l'Académie bulgare des sciences, 2, Saborna, 1000-Sofia, Bulgarie nikolaysirakov@gmail.com

(2) Université Bordeaux 1, UMR 5199 du CNRS, Institut de Préhistoire et de Géologie du Quatemaire, PACEA, Avenue des Facultés, 33045 Talence, France - ttsenka@yahoo.fr 


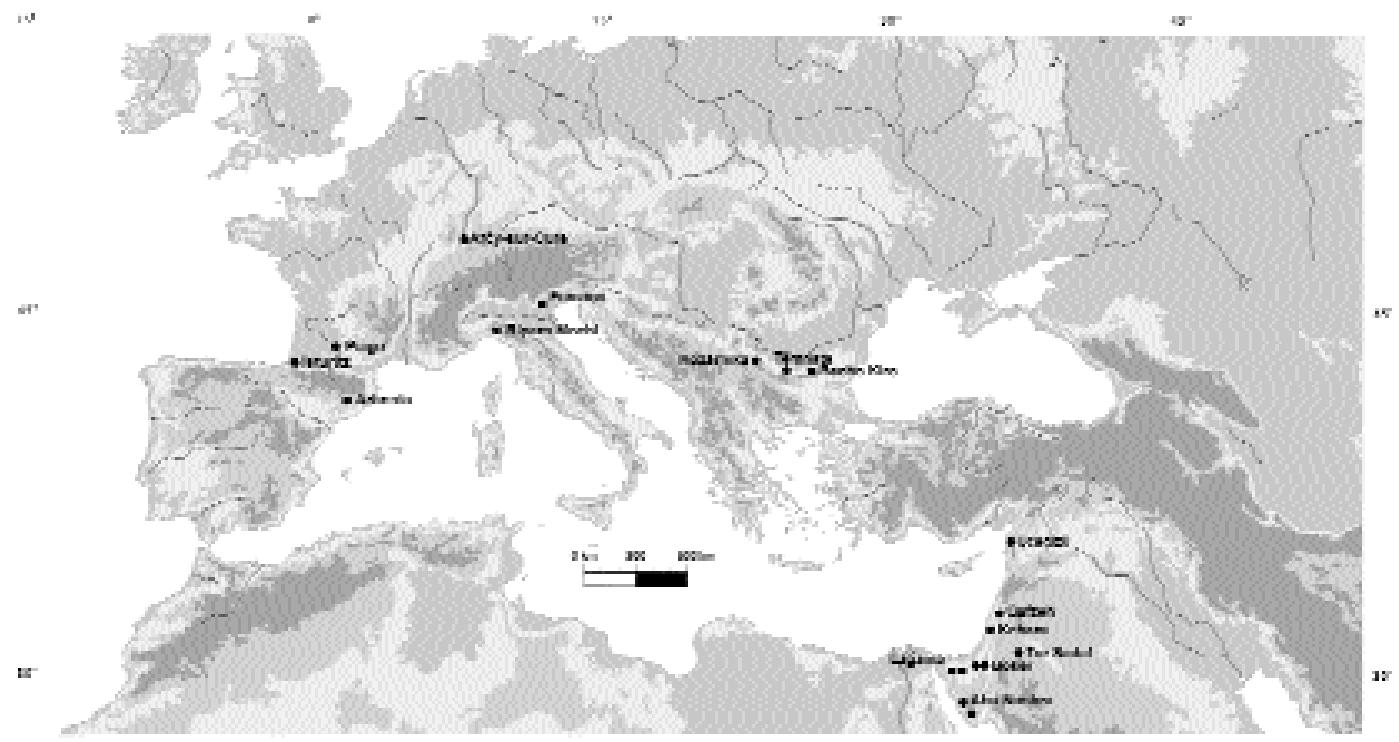

Figure 1 - Localisation de la grotte Kozarnika, Temnata, Bacho Kiro et des sites du début du Paléolithique supérieur en Europe et au Proche-Orient, mentionnés dans le texte.

Figure 1 - Location of Kozarnika, Temnata and Bacho Kiro caves and others mentioned Early Upper Palaeolithic sites in Europe and Middle East.

\section{LE SITE}

La grotte Kozarnika découverte en 1931 par le préhistorien bulgare Rafaïl Popov (Popov 1933) est localisée dans le nord de la partie occidentale des Pré-Balkans près de la plaine danubienne, à environ $60 \mathrm{~km}$ au sud du Danube et à quelques $30 \mathrm{~km}$ de l'actuelle frontière serbe (fig.1).

La grotte se situe à une altitude de $481 \mathrm{~m}$ et l'entrée est ouverte vers le sud sur le versant nord d'une vallée, dans les escarpements calcaires du Jurassique supérieur, à la limite de la plaine danubienne. Fouillée depuis 1996, la grotte de Kozarnika constitue la troisième séquence importante de l'est des Balkans, après Temnata et Bacho Kiro, situées au point de départ de la voie danubienne.

\section{LA SÉQUENCE DU PALÉOLITHIQUE SUPÉRIEUR: POSITION CHRONO-STRATIGRAPHIQUE DU NIVEAU VII}

Le matériel lithique décrit ici provient d'un dépôt archéologique découvert sur une surface de $18 \mathrm{~m}^{2}$ et d'une épaisseur comprise entre 5 et $20 \mathrm{~cm}$. Ce niveau VII, fouillé durant trois campagnes de terrain $(1998,2001$ et 2002) a livré plus de 6000 objets lithiques (nombreux débris et autres déchets exclus).

Son contexte est présenté ci-dessous dans la séquence des unité sédimentologiques et niveaux archéologiques.

La séquence entière de Kozarnika, épaisse d'environ $8 \mathrm{~m}$, présente un dépôt du Paléolithique supérieur d'environ $1,5 \mathrm{~m}$. Les processus géologiques dominants sont marqués localement par une forte cryoturbation. Le développement d'un pergélisol dans la grotte pendant le
Paléolithique supérieur est à l'origine des déformations des couches (Ferrier et Leblanc 2004). Cependant, dans le vestibule, il y a des secteurs mieux préservés, où l'on pouvait établir une archéoséquence et dont les occupations ont été assez intensives pour s'exprimer par une succession de niveaux d'habitat, bien marqués et séparables à certains endroits.

Les unités litho-sédimentologiques sont identifiées par une numérotation arabe, tandis que les ensembles archéologiques sont indiqués en chiffres romains.

La stratigraphie de la séquence du Paléolithique supérieur est la suivante, en lisant de haut en bas (Guadelli et Sirakov 2004) :

- les niveaux archéologiques I-III (couches géologiques 3a et 3b) ont livré des industries lithiques à pointes et lamelles à dos appartenant aux stades récents du Gravettien local. Les dates ${ }^{14} \mathrm{C}$ sur charbon de bois ont donné pour la couche $3 \mathrm{a}$ : $11490 \pm 120$ BP (Gif-109911/GifA-98346) et $11550 \pm 100$ BP (Gif-10990/GifA-98345) et pour la couche 3b : $19770 \pm$ 270 BP (Gif-10674) et $19890 \pm 270$ BP (Gif-10673) ;

- le niveau archéologique IV (couche géologique 4 fortement marquée par des processus de cryoturbation, de comblements de fentes de gel et blocs effondrés) a livré une industrie lithique attribuée à une phase moyenne du Gravettien balkanique, datée de $26010 \pm 270$ BP (Gif10676/GifA-97286) et $26120 \pm 120$ BP (Gif/LSM-10677), qui se caractérise par la présence de lamelles à dos et par un type spécifique de pointes larges et rectilignes, très proches de l'idée de fléchettes - les pointes de type Kozamika (Tsanova 2003) ; 


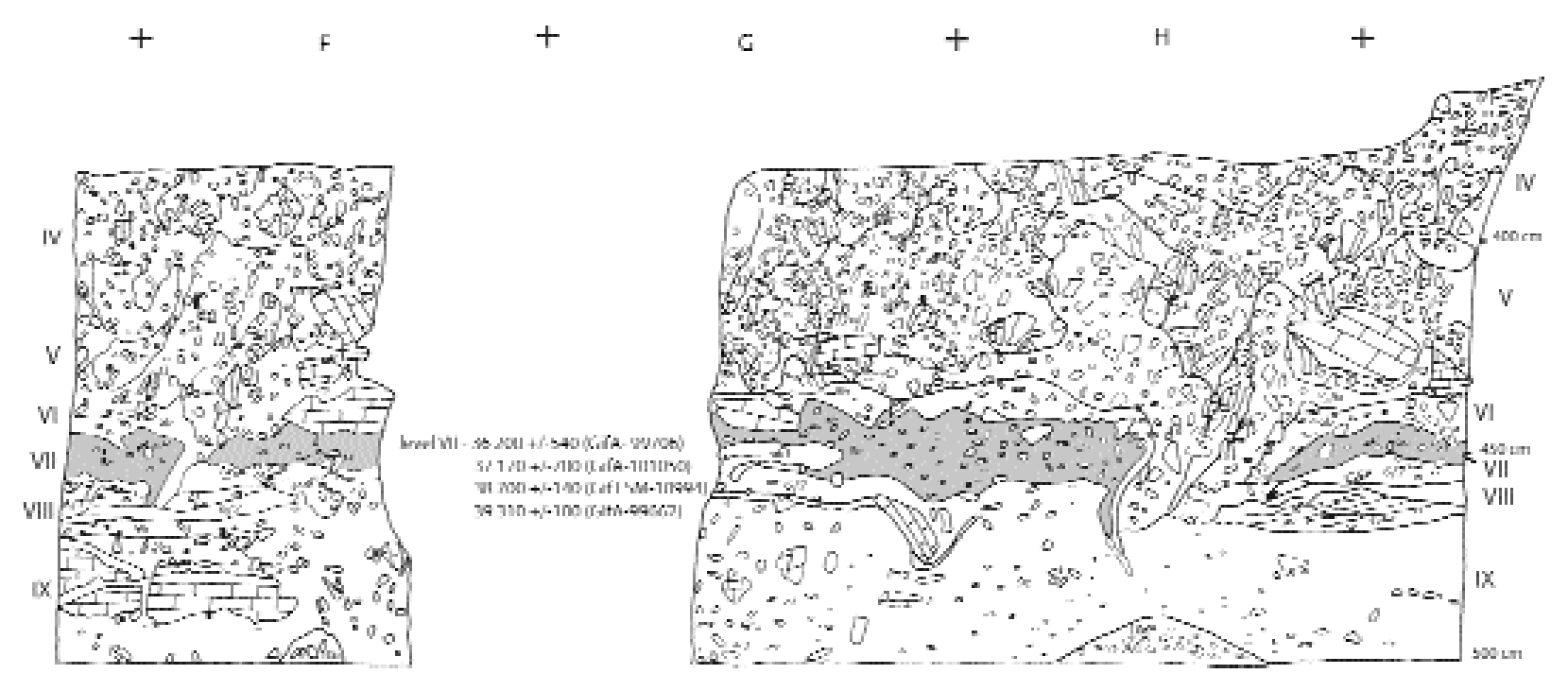

Figure 2 - Coupe transversale nord, ligne F-H entre les secteurs 7/8, position chrono-stratigraphique et datations C14 du niveau VII de la grotte Kozarnika.

Figure 2 - Northern transversal section, row F-H, between sectors 7\&8. Chronological position and 14C dates of layer VII at Kozarnika.

- le niveau $\mathrm{V}$ (couche sédimentologique $5 \mathrm{a}$ ), daté de 25 $650 \pm 730$ BP (Gif-10992) et le niveau VI (couche sédimentologique $5 \mathrm{~b}$ ) daté de $26490 \pm 270$ BP (GifA-99044) ont livré des industries lithiques lamellaires à pointes à dos minces. Ces niveaux inédits ne contiennent pas de pointes de type Kozarnika et ils semblent plutôt proches des niveaux sous-jacents.

La lacune chrono-sédimentologique de 10000 ans au moins entre les niveaux VI (26 Ka BP) et VII (36 Ka BP) reste problématique parce que, d'une part elle ne correspond pas à une érosion détectable et, d'autre part, elle est trop longue pour un arrêt de la sédimentation.

- Le niveau VII (contenu dans le sédiment $5 \mathrm{c}$ et appelé aussi "VI inférieur" dans les rapports de fouilles) se place dans la partie basale de la séquence du Paléolithique supérieur (fig.2). La couche $5 c$ est constituée d'un limon brun foncé contenant de nombreux petits cailloux de moins de $3 \mathrm{~cm}$, des graviers de calcaire principalement et des silex. Les sédiments sont riches en os brûlés et charbons de bois. La limite supérieure de la couche est nette et déformée par la cryoturbation. Pour le niveau VII, il y a une série cohérente de dates ${ }^{14} \mathrm{C}$ entre $36 \mathrm{Ka}$ et $39 \mathrm{Ka} \mathrm{BP}$ (fig.2) ;

- la couche géologique sous-jacente $6 / 7$, localisée à la limite entre la séquence moustérienne et le Paléolithique supérieur, contient deux phases de niveau archéologique VIII. L'industrie lithique de la couche $6 / 7$ a livré deux ensembles technologiques distincts : l'un Levallois et l'autre lamellaire identique à l'ensemble étudié du niveau VII. Le faible effectif du matériel lithique de la couche $6 / 7$ ne permet pas, pour l'instant, une analyse poussée. En attente d'une caractérisation plus précise de l'origine de cette composition, nous avons préféré porter l'attention sur le niveau VII, répandu sur tout l'espace exploré et lié à des foyers et aux nappes successives de vestiges, nettement individualisées dans certains carrés. II faut souligner que, sauf une partie de phalange (couche 6/7), aucun autre reste humain n'a été découvert jusqu'à présent dans le contexte des ensembles du début de Paléolithique supérieur de Kozamika ;

- la séquence moustérienne contenue dans les couches sédimentologiques 9 et 10 se caractérise par un débitage préférentiel centripète ou bipolaire, réalisé selon le concept Levallois. La partie inférieure de la séquence du Paléolithique moyen (couche 10), datée entre 200-130 Ka renferme des pièces à retouche bifaciale tandis que la partie supérieure (couche 9), plus récente que $130 \mathrm{Ka}$ livre quelques pointes foliacées bifaciales. Pour les deux couches moustériennes, les formes retouchées les plus fréquentes sont des éclats retouchés et denticulés, des racloirs, dont la plupart latéraux. Les pointes moustériennes sont rares (Guadelli et al. 2005).

\section{DIAGNOSTIC TECHNO-TYPOLOGIQUE DU NIVEAU VII}

Ce premier diagnostic qualitatif a été établi à partir d'une série d'environ 3000 objets lithiques qui constitue la moitié du matériel. Cette série provient de $6 \mathrm{~m}^{2}$ (secteurs 9 et 10). Menées en parallèle de la monographie du site, deux thèses de doctorat sont en cours : une concernent le 

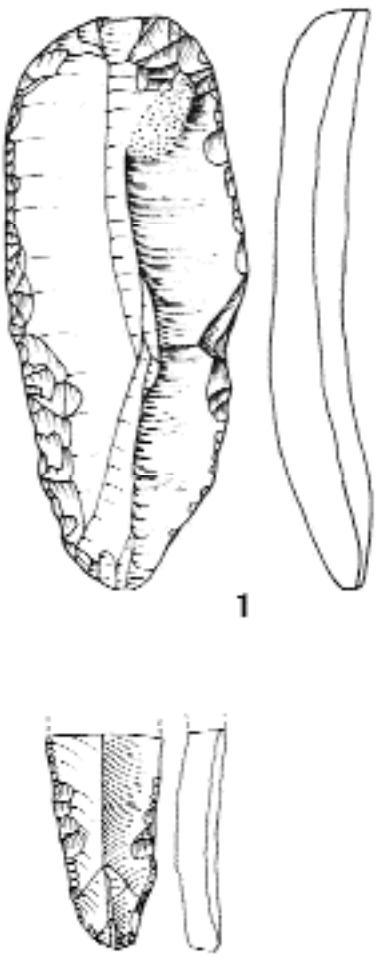

4
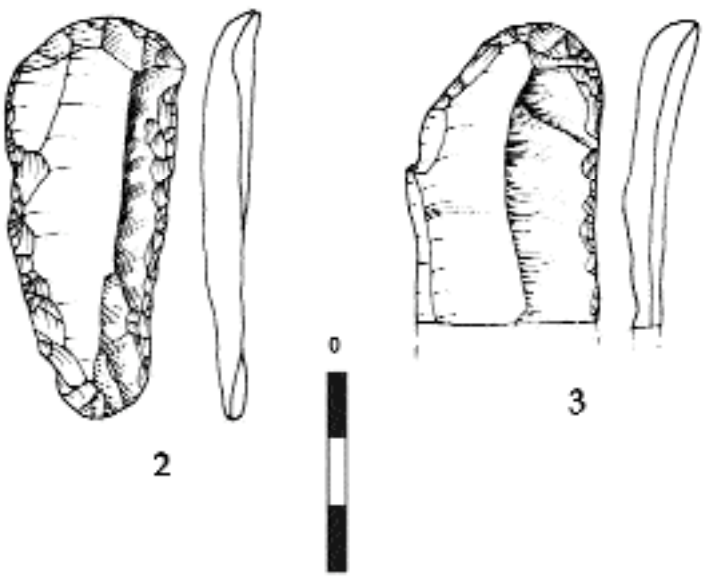

3

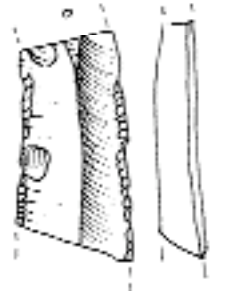

5

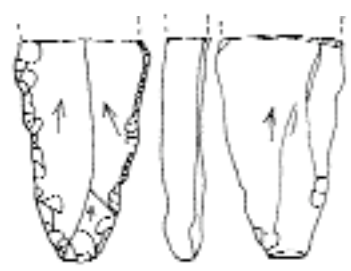

6
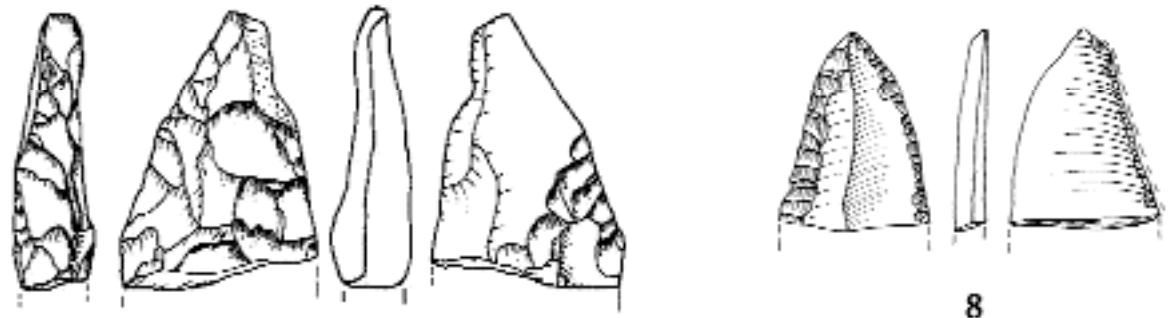

8
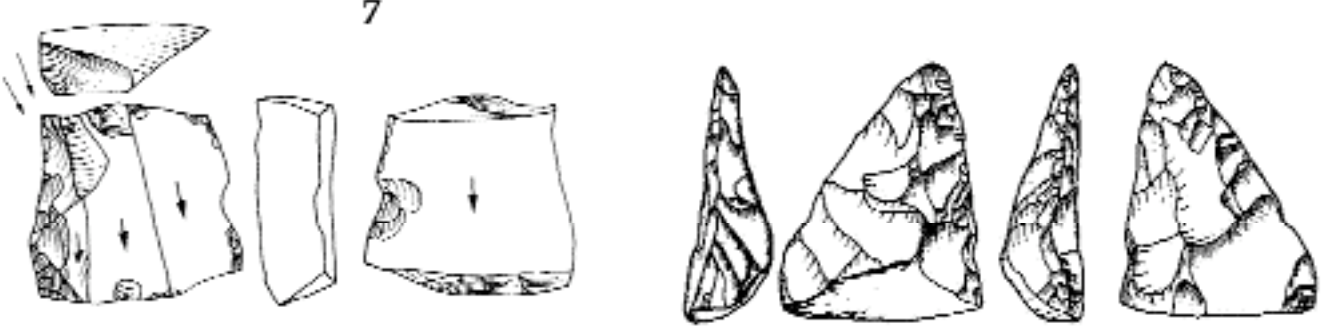

9

10

Figure 3 - Kozarnika, industrie lithique du niveau VII. Outils du fond commun : 1-3 grattoirs sur lames. Remarquer la largeur importante des lames et les profils légèrement arqués, les faces supérieurs à négatifs unidirectionnels parallèles ; 4-6

Fragments de lames étroites retouchées. Remarquer lames 4 et 6 issues d'un débitage unipolaire convergent, tandis que la lame 5 dérive d'un débitage bipolaire ; 8 Lame appointée ; 7, 10 Pièces à retouche bifaciale ; 9 Burin.

Figure 3 - Kozarnika, layer VII, lithic industry. Common tools : 1-3 End scrappers on blade, Note the sizeable width, the slightly curved profile of the blades and the dorsal faces with unidirectional and parallel scares of previous taken off blades. 4-6:

Fragments of narrow retouched blades. 4 and 6 come from unipolar convergent debitage, 5 comes from bipolar debitage. 8 : pointed blade. 7, 10 : pieces with bifacial retouch. 9: burin. 
matériel lithique du Paléolithique supérieur (S. Taneva AIM-BAS, Sofia) et l'autre l'industrie osseuse (A. Guadelli AIM-BAS, Sofia). L'étude complète du matériel lithique contenu dans le niveau VII (de secteurs 9 et 10) a été effectuée dans le cadre de la thèse de doctorat de Ts. Tsanova (2006).

\section{LES MATIÈRES PREMIÈRES}

L'état de la recherche sur les matières premières n'a pas pe mis de caractériser précisément leur économie. Malgré cela, environ $90 \%$ des silex utilisés par les hommes préhistoriques de Kozarnika sont d'origine locale. Dans les autres cas, la matière première a probablement une origine plus éloignée mais il est aussi possible que certains types soient des variantes rares du silex local.

Dans le dépôt primaire (les calcaires du Jurassique supérieur), ainsi que dans les parois de la cavité, le silex se tro uve sous la forme de nodules aplatis et tectonisés (Natchev 2003), inaptes à la taille.

Dans les dépôts secondaires (éluviaux, colluviaux, alluviaux), le silex se trouve sous la forme de petits nodules $(4-7 \mathrm{~cm})$, de blocs et de fragments gélifractés. La matière première des dépôts secondaires est de meilleure qualité que celle provenant des gîtes primaires.

L'homme préhistorique de Kozarnika a dû vraisemblablement se servir des dépôts secondaires de silex. Les travaux sur les sources de silex plus lointaines sont en cours et on pense que certaines variétés de silex brun-jaune " balkanique" proviennent probablement de gîtes localisés autour de 80 - $100 \mathrm{~km}$ au sud, dans la région de Vratsa.

\section{INDUSTRIE LITHIQUE}

Le débitage lamellaire dans le niveau VII est effectué essentiellement sur la matière première locale. Un approvisionnement plus lointain est observable seulement pour quelques lames et esquilles de retouches.

Le débitage lamellaire a été pratiqué sur des nodules de petites dimensions $(3-5 \mathrm{~cm})$ et sur des plaquettes ou autres fragments gélifractés. Une autre modalité de débitage lamellaire s'exerce sur des éclats épais.

\section{LE DÉBITAGE DES LAMES}

L'absence de nucléus à lames ne permet pas la reconsti tution précise du mode de production laminaire. Toutefois, les quelques lames entières témoignent d'un schéma de débitage unipolaire, intercalé, à partir d'une table laminaire large et relativement plane (fig.3: $1-3$ ).

Pour une partie des lames, les stigmates (lèvres prononcées, absence de marques d'impact, bulbe diffus ou absent) de la percussion au percuteur tendre organique (Pelegrin 2000) sont nets et il ne fait aucun doute que les préhistoriques du niveau VII de Kozarnika ont maîtrisé cette technique.

La plupart (environ 2/3) des lames (au total 30 exemplaires) sont en silex non local (par ex. brun-jaune) et de rares variétés, en silex locaux de meilleure qualité. L'absence des nucléus correspondant à ces lames et la présence des éclats de retouches en matières premières non locales indiquent qu'une partie de la production laminaire a été probablement importée et retouchée dans la grotte.

Ces lames, relativement plus grandes et larges en silex non local ont été utilisées pour la fabrication d'une petite quantité de grattoirs, lames retouchées ou/et appointées par une retouche directe et relativement envahissante (fig.3 : 4-6, 8).

\section{DÉBITAGE LAMELLAIRE}

\section{Les techniques}

La plupart des nucléus à lamelles présentent des plans de frappe lisses ou avec des reprises partielles. Les bords des plans de frappe, dans quelques cas émoussés, témoignent d'une préparation soignée par abrasion. Les contrebulbes ne sont pas marqués. La détermination des techniques d'enlèvement n'est pas très nette, d'autant plus que les talons des lamelles brutes sont minces et les stigmates ne sont pas très lisibles. Néanmoins, une bonne partie des supports lamellaires minces témoigne d'un geste tangentiel utilisant soit la pierre tendre, soit un percuteur organique.

Une chaîne opératoire principale unipolaire pyramidale et semi-toumante est destinée à la production systématique des lamelles (fig.4). Un deuxième schéma de débitage (huit nucléus, une lamelle retouchée et cinq lamelles brutes) est bipolaire semi-tournant à partir des nucléus prismatiques. Une troisième modalité, qui pourrait être la variabilité d'un même concept de débitage, est établie sur la tranche du support (fig.5).

\section{DÉBITAGE UNIPOLAIRE A LAMELLES}

Le schéma de débitage le plus fréquent est unipolaire semi-toumant et réalisé par une mise en forme des parties postérieures et inférieures du nucléus (fig.6 : 1, 4). Le plein débitage commence à partir d'un volume de type pyramidal, irrégulier, aux arêtes convexes, avec un plan de frappe incliné (environ $40-60^{\circ}$ ). Ce plan de frappe est entretenu par des enlèvements de tablettes partielles (fig.4).

Les lamelles obtenues sont étroites et pointues (à bords convergents), à profil légèrement courbe, surtout dans la partie distale et témoignent d'enlèvements qui outrepassent les tables lamellaires. Les aménagements de la partie inférieure (crête inférieure) du nucléus prédéterminent la forme appointée des lamelles.

\section{DÉBITAGE BIPOLAIRE A LAMELLES}

Le deuxième schéma de débitage, moins fréquent, est bipolaire, semi-tournant. II nous semble que, dans la plupart des cas, on ne puisse pas parler d'un véritable débitage bipolaire c'est-à- dire réalisé d'après un projet, mais plutôt d'un aménagement de la surface (accidents, cintrage). 

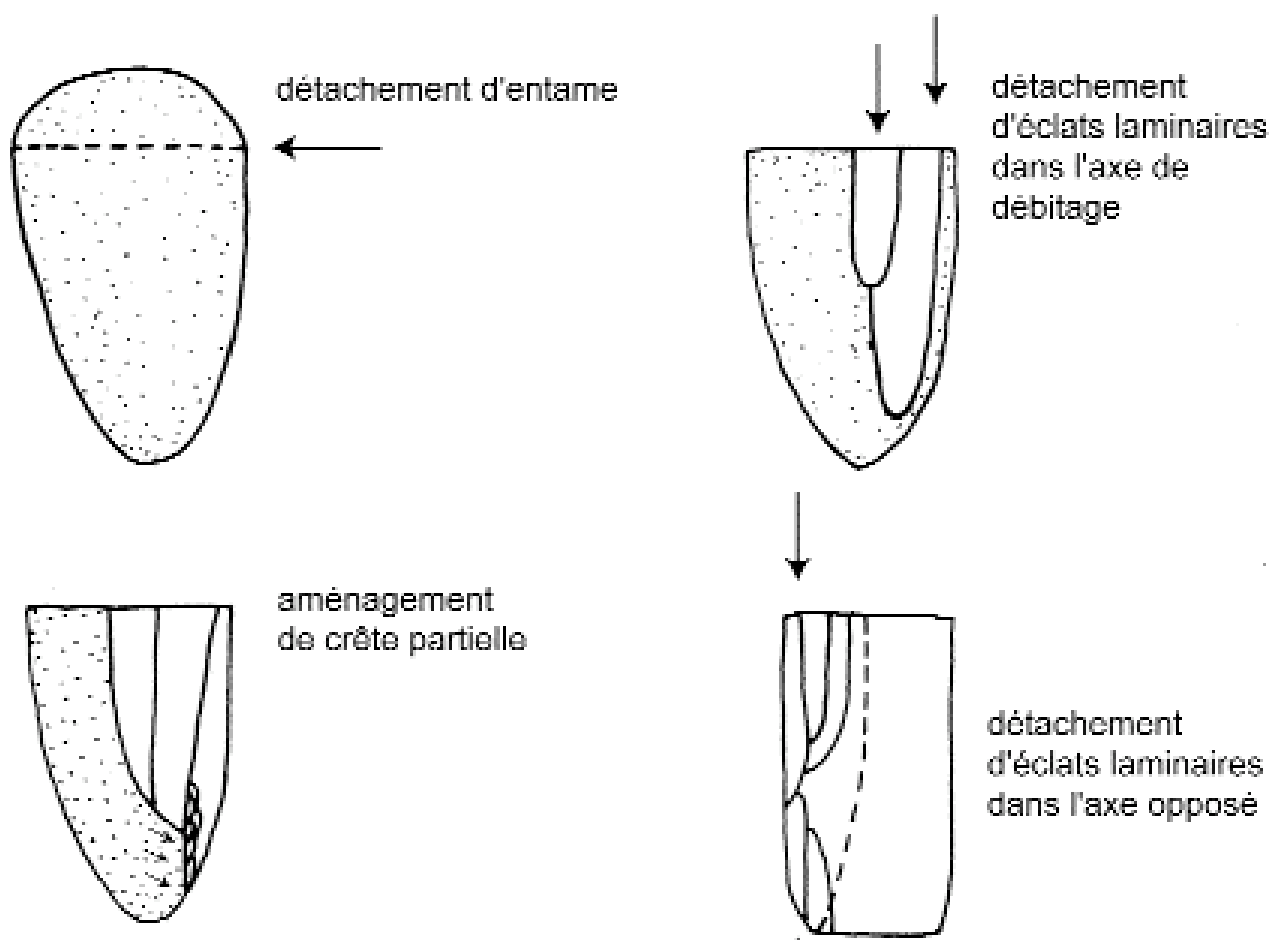

enlèvements des éclats de flanc:
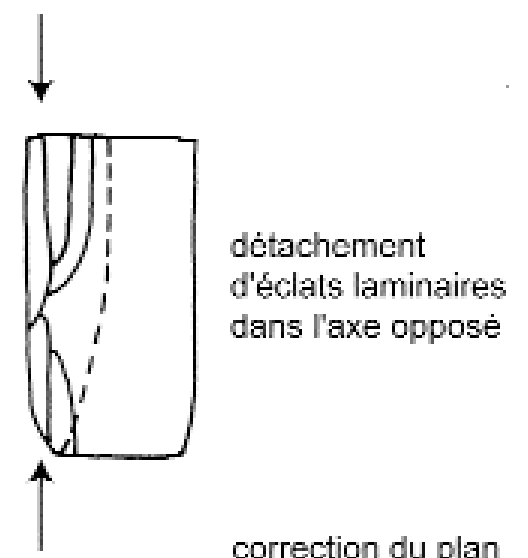

dètachement

d'éclats laminaires dans l'axe opposé entretiens du cintrage de frappe
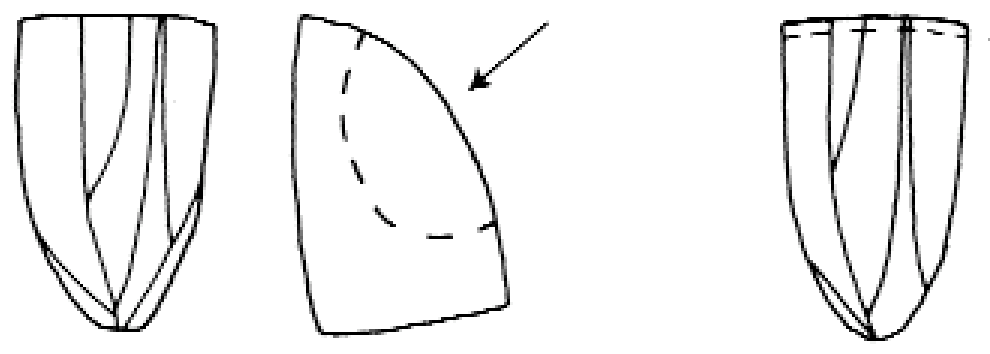

enlèvement

de tablette entière ou partielle

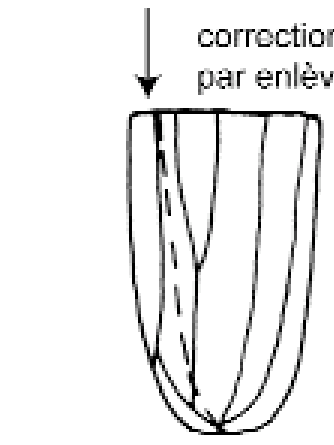

Tevose n?

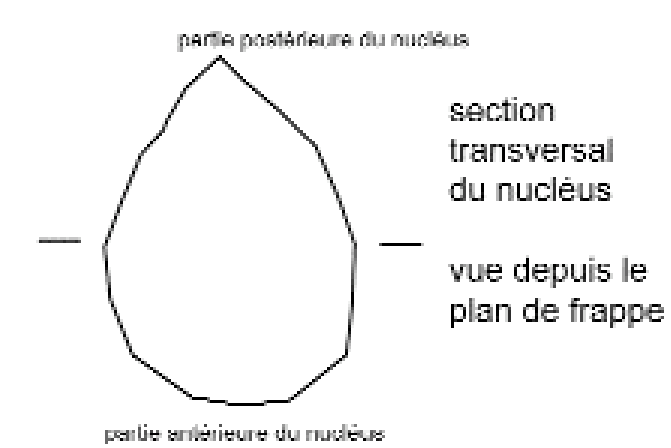

Figure 4 - Kozarnika, niveau VII : Reconstruction schématique de débitage unipolaire et bipolaire lamellaire.

Figure 4 -Kozarnika, Layer VII. Unipolar and bipolar microblade production, schematic reconstitution. 


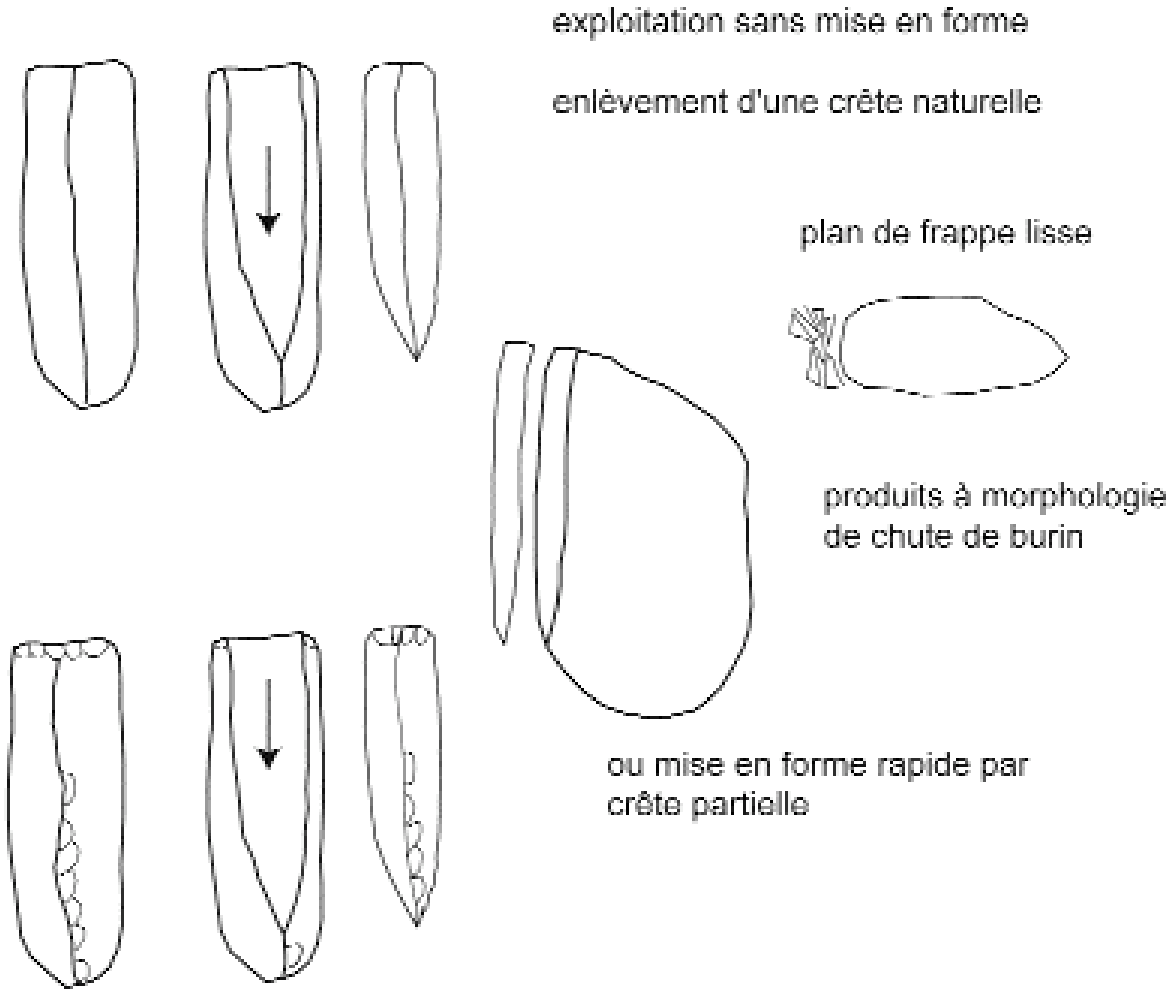

Figure 5 - Kozarnika, niveau VII : Reconstruction schématique de débitage sur la tranche du support.

Figure 5 - Kozarnika, Layer VII. Debitage from the edge of the blank, schematic reconstitution.
T5 $0 S$

Toutefois, les quelques exemplaires montrent des surfaces de débitage planes. L'entretien de ces nucléus prismatiques bipolaires se fait par des réaménagements latéraux (des crêtes et néocrêtes à un versant préparé vers la partie postérieure du nucléus (fig.6 : 4). Les lamelles brutes issues de ce schéma bipolaire sont plus larges que les lamelles de débitage unipolaire. Seules cinq lamelles brutes sont à profil rectiligne et bords parallèles. Une lamelle provenant de débitage bipolaire est retouchée.

\section{DÉBITAGE SUR TRANCHE DU SUPPORT}

Une autre modalité de débitage est effectuée sur la tranche d'un support plat (éclat, plaquette ou bâtonnet gélifracté). Ce débitage lamellaire peut commencer par l'enlèvement d'une crête " naturelle ". Parfois, on observe une mise en forme rapide par l'aménagement d'une crête partielle, rectifiant l'arête centrale d'ouverture du débitage. La remise en forme de ces nucléus se fait par l'aménagement d'une néocrête partielle. Une partie de la production lamellaire issue de ces éclats et plaquettes gélifractées aura la morphologie de chutes de burin (fig.5).

\section{DISCUSSION SUR LES PRODUITS RECHERCHÉS ET LA TRANSFORMATION EN OUTILS}

Les produits recherchés sont des lamelles étroites,certaines pointues (à bords convergents), destinées à la production d'un type de pointes par fine retouche directe bilatérale, abrupte vers la partie proximale de la lamelle et plus fine, voire parfois marginale, vers la partie mésio-distale. Cette retouche bilatérale peut être discontinue dans la zone mésiale, ce qui introduit une modification légère de morphologie des bords parallèles qui deviennent légèrement arqués (fig.7). De la faible présence de spécimens entiers, résulte une moins forte visibilité de ce type de pointes. II est fort probable qu'une partie des lamelles à dos mince soient des fragments proximaux et mésiaux de ces pointes.

Dans le cadre des études préliminaires sur les outils diagnostiques du niveau VII, l'équipe de notre projet a eu la possibilité d'inviter M. Lenoir et $\mathrm{G}$. Lucas pour leur montrer des échantillons représentatifs de ce matériel. A cette occasion, nous avons pu discuter sur l'identification culturelle de l'ensemble en confrontant nos hypothèses avec les avis plus " neutres " des collègues extérieurs à l'équipe. En les remerciant, nous présentons ci-dessous leurs opinions.

L'un de ces rapports décrit les outils diagnostiques comme:

"...des lamelles fines allongées à retouche marginale bilatérale, appointées " (Lenoir 2001).

L'autre étude, beaucoup plus poussée et approfondie, argumente la possibilité de rattacher le niveau VII à l'origine du Gravettien (Lucas 2000). Une comparaison morphologique et dimensionnelle confronte les lamelles à dos mince du niveau VII aux pointes à dos de Corbiac, Roc-deCombe, Le Flageolet I et Tercis : la moyenne des largeurs $(8,82 \mathrm{~mm})$ est dans la fourchette de 6,35 à $11,5 \mathrm{~mm}$, tandis que la moyenne de l'épaisseur $(2,4 \mathrm{~mm})$ pour les 


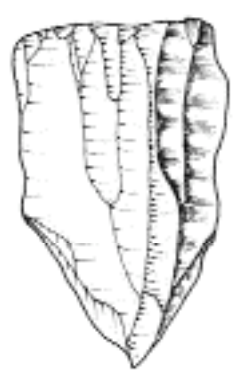

1

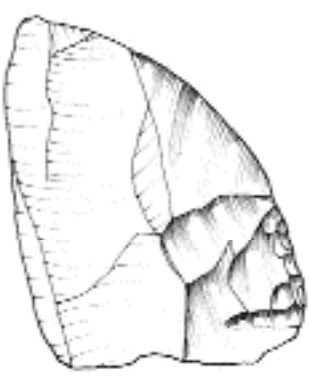

D

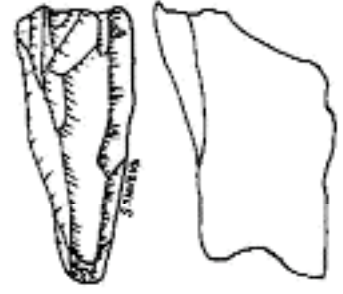

2

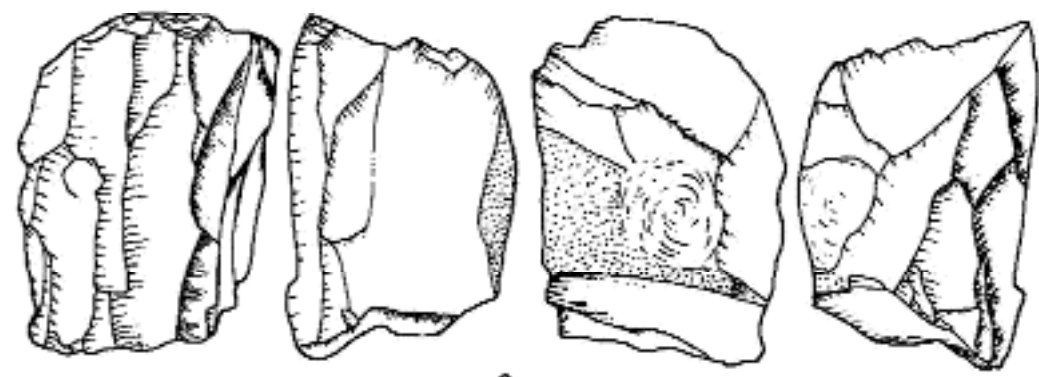

3

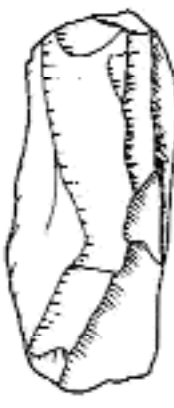

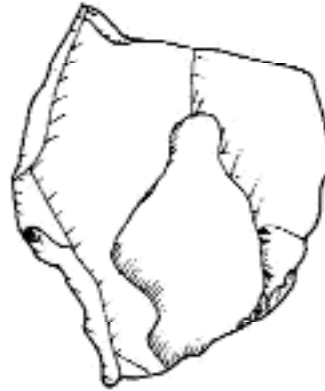

4
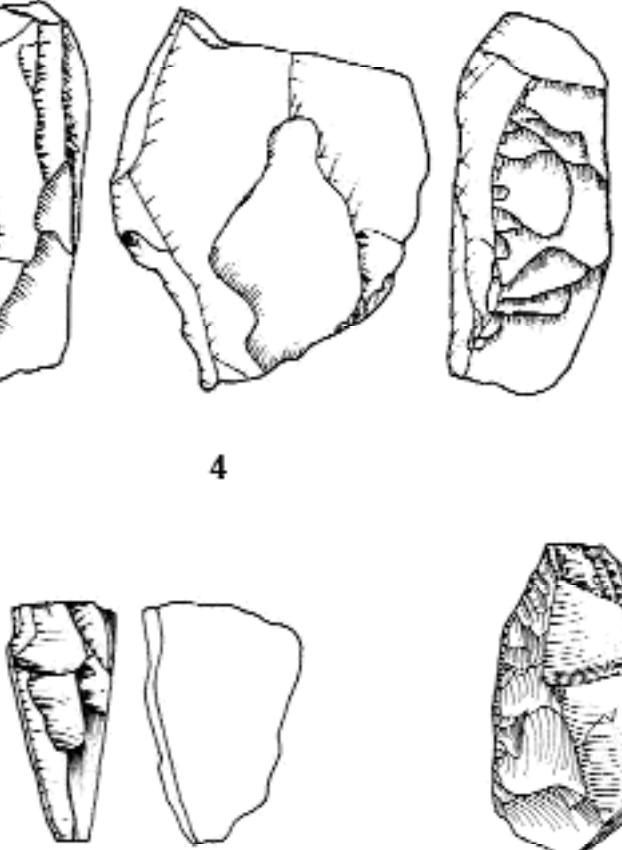

6

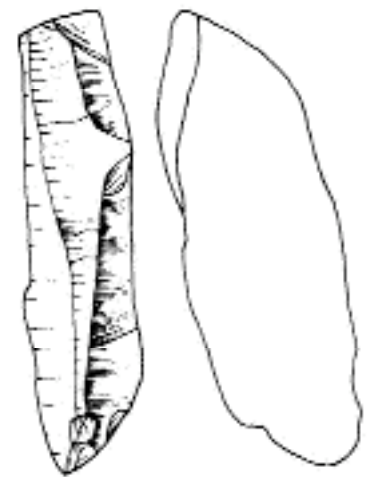

5
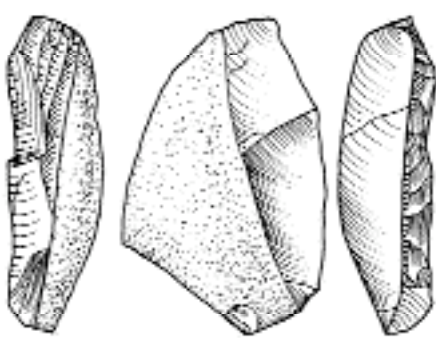

7

Figure 6 - Kozarnika, industrie lithique du niveau VII. Nucléi : 1 Nucléus unipolaire prismatique. Remarquer la crête postérieure qui aménage les surfaces latérales larges, aussi le négatif d'aménagement du bord inférieur qui appointe le nucléus et prédétermine la forme pointue de lamelles. 2, 5, 6 : Nucléi sur tranche du support : débitage de lamelles sur l'épaisseur d'éclat ou plaquette gélifractée; 3 Nucléus prismatique bipolaire ; 4 Nucléus bipolaire sur la face longue et étroite d'une plaquette gélifractée. Remarquer la crête postérieure à un versant ; 7 Fragment de nucléus à lamelles sur support cortical, modifié en outil (racloir?) ou remise en forme.

Figure 6 - Kozarnika, layer VII, lithic industry. Cores: 1: unipolar prismatic. Note the posterior crest producing large lateral surfaces and the traces of shaping of the lower part of the core inducing the pointed outline of bladelets. 2,5,6: Cores on blank's edge, bladelet production on flakes or frozen slab. 3 Bipolar prismatic core; 4:Bipolar core made on the long and narrow face of a frozen slab. Note the one side posterior crest. 7 Core fragment of a bladelet core on cortical blank. 

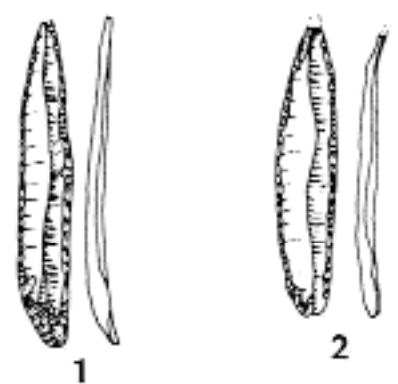

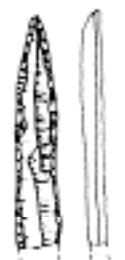

3
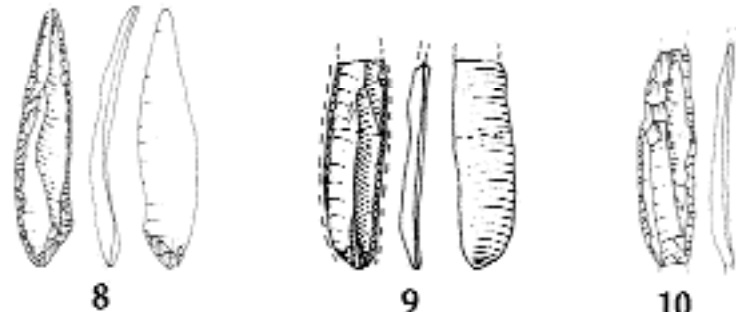

10

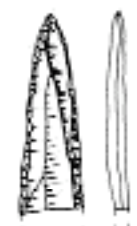

4

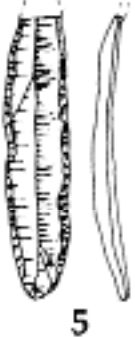

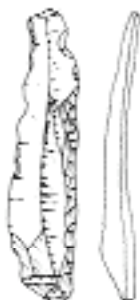

11

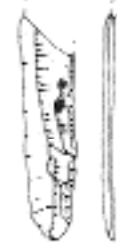

16

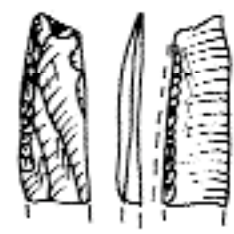

21

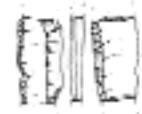

25

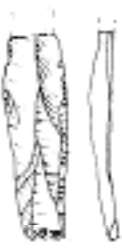

12

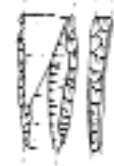

17

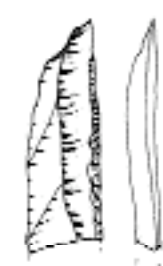

13

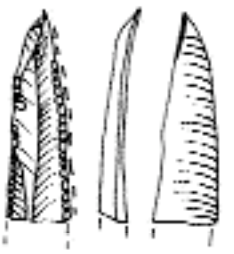

14

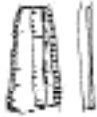

19

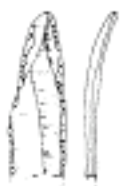

15

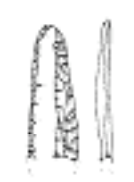

18
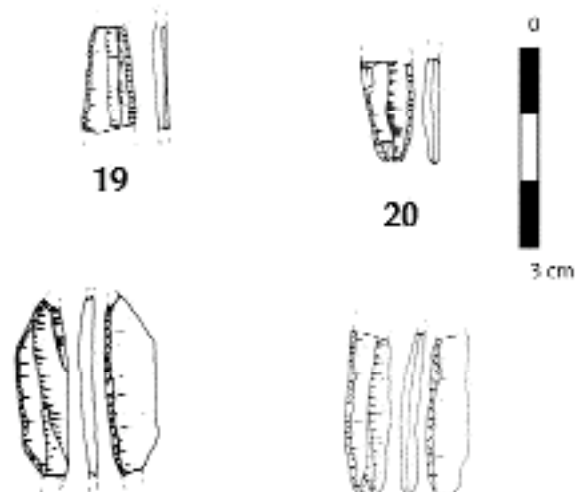

23

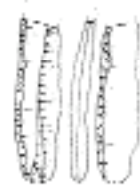

24

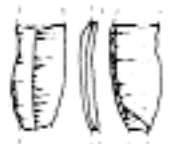

26

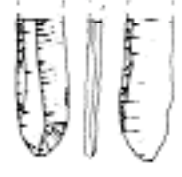

27

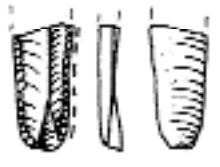

28

Figure 7 - Kozarnika, industrie lithique du niveau VII. Outils diagnostiques : 1-10;12, 14-16;18-20 Pointes et lamelles à dos mince ; 11, 13, 17 Lamelles à dos épais 21-27 Lamelles à retouche alterne ; 28 Lamelle retouchée.

Figure 7 - Kozarnika, layer VII, lithic industry. Diagnostic artefacts 1-10, 14-16, 18-20; Pointes and bladelets with thin back; $11,13,17$ bladelets with thick back, 21-27 bladelets with alternate retouch; 28 Retouched bladelet. 
lamelles de Kozarnika est nettement au-dessous de 3,30 $\mathrm{mm}$ à $4,88 \mathrm{~mm}$. II faut souligner que les pointes et lamelles à dos épais sont de largeur plus réduite puisque la retouche abrupte est sensée enlever plus de matière, tandis que les pointes à dos mince sont très peu modifiées par la retouche. Par conséquent, il est évident que dans le Gravettien français, les supports de pièces à dos sont plus robustes que ceux de Kozamika.

D'après G. Lucas, les lamelles à dos sont atypiques puisque la retouche est fine et semi-abrupte. L'aspect de cette retouche semi-abrupte est en relation avec la matière première puisqu'elle se trouve sous la forme de petits rognons et de plaquettes gélifractées. D'après nous, ce constat est peu probable parce que dans les niveaux susjacents les lamelles à dos sont produites à partir des mêmes modules de blocs. Cependant, les dos sont clairement plus abrupts. Ceci plaide en faveur de l'intention de produire des lamelles plus épaisses qui s'accentuent dans les couches sus-jacentes (niveau VI, V, IV). Des lamelles proches de celles du type gravettien à dos abrupt et semiabrupt sont au nombre de 22 sur un total de 51 pièces à dos. La majorité des cas (nb 46) sont à dos mince. Cinq dos seulement sont épais (Lucas 2000).

Dans les niveaux sus-jacents $\mathrm{VI}$ et $\mathrm{V}$, ces lamelles à dos mince coexistent avec les lamelles à dos épais (Lenoir 2001). C'est dans le niveau IV b (daté d'environ $26 \mathrm{Ka}$ $\mathrm{BP})$ que les lamelles à dos mince diminuent fortement et les outils diagnostiques apparaissent nettement plus variés : pointes de type Kozarnika, pointes gravettiennes de petites dimensions à dos semi-abrupt et $a b$ rupt, lamelles à dos et lamelles à dos tronquées (Tsanova 2001).

\section{LES OUTILS DU FOND COMMUN}

Les lames relativement plus larges $(15-40 \mathrm{~mm})$ dont certaines en matière première non locale (dont les nucléus sont absents) ont été utilisées pour la fabrication des grattoirs (fig. $3: 1-3$ ) ou des lames retouchées (fig.3 : 4-6). Ces grattoirs à retouche latérale, parfois envahissante, rappellent des grattoirs à front bas, décrits par $D$. de Sonneville-Bordes et Perrot (Sonneville-Bordes et Perrot 1954) et, plus récemment, par Demars et Laurent (1992) comme abondants dans l'Aurignacien mais, en réalité, c'est une forme omniprésente. Quelques lames appointées présentent aussi une retouche fine et inverse (fig. $3: 8$ ). Les perçoirs et les troncatures sont sporadiques. Les burins, dans le sens typologique du terme, sont quasi absents (fig. $3: 9$ ).

Deux pièces à retouche couvrante et bifaciale (fig. $3: 7,10$ ), fabriquées en matière première locale évoquent " des souvenirs moustériens " et rappellent des pointes foliacées bifaciales typiques du Moustérien sous-jacent de la couche 9. Dans ce niveau, nous constatons une absence totale de " fossiles directeurs " typiques de l'Aurignacien ancien et des phases plus récentes de l'Aurignacien. II n'y a pas de grattoirs carénés et à museau, ni de véritables lames auri- gnaciennes appointées ou étranglées, ni de petites lamelles courbes ou torses - de type Dufour. L'industrie en os est peu abondante, sans forme diagnostique de l'Aurignacien.

\section{SYNTHÈSE}

\section{1 - Diagnostic}

Le débitage lamino-lamellaire du niveau VII de Kozamika est effectué par percussion tendre, à partir d'un volume pyramidal ou prismatique. Le débitage lamellaire a pour objectif la production de lamelles pointues (à bords convergents) et de lamelles à bords parallèles. Les premières sont destinées à la production de pointes à dos mince à retouche marginale bilatérale appointant (fig.7 : 1$10,12,15,16,18-20)$, rarement de pièces à dos un peu plus épais (fig.7 : 11, 13, 17) et les deuxièmes sont à retouche alterne fine (directe et inverse) (fig.7 : 16-27) dont certaines très proches de celle de grandes lamelles Dufour rectilignes, comparables à celles du Piage, dans la couche $\mathrm{K}$ (Bordes 2002). Les produits recherchés pour le débitage laminaire sont des lames larges $(20-30 \mathrm{~mm})$ à profil rectiligne ou légèrement courbe (fig.3: 1-3).

La forte fragmentation des lamelles retouchées ne permet pas de percevoir une variabilité nette et individualisée (par exemple : $y$-a-t-il des pointes à retouche inverse?).

\section{2 - Comparaisons}

A la suite de récents travaux sur les industries lamellaires anciennes du Proche-Orient (Goring-Morris and Belfer-Cohen 2003 ; Otte et Kozlowski 2004), sur la redéfinition des premières phases de l'Aurignacien en E u rope (Bon 2000 ; Bordes 2002 ; Teyssandier 2003) et la révision du Bachokirien qui apparaît comme une industrie de transition entre le Paléolithique moyen et le Paléolithique supérieur et non plus comme une origine de l'Aurignacien (Rigaud et Lucas 2002, 2006 ; Rigaud 1998, 2001 ; Teyssandier 2003 ; Tsanova et Bordes 2003), nous souhaitons apporter ici quelques éléments de réflexion concernant le début du Paléolithique supérieur dans les Balkans et plus particulièrement à travers l'étude du débitage lamellaire.

Les schémas de débitage et les outils diagnostiques associés (les pointes et lamelles à dos fin et celles à retouche alterne et inverse) dans leur contexte chrono-stratigra phique, permettent de rapprocher le Kozarnikien des faciès contemporains du Proche-Orient, tel que l'Ahmarien ancien. Les sites de Qafzeh, Kebara, Boker A et BE, Lagama VIII et VII, Abu Hoshra I, II et Tor Sadaf (fig.1) ont livré des industries à débitage lamellaire, datées entre 38/36-26 Ky (Belfer-Cohen and Goring-Morris 2003). Ces ensembles se caractérisent par des lamelles appointées à retouche fine bilatérale directe, issue d'un débitage unipolaire volumétrique. Les pointes el-Wad issues d'une même conception de débitage portent de fines retouches bilatérales, directes ou inverses (Monigal 2003). 
Dans le site de Tor Sadaf, deux ensembles sous-jacents à un niveau dit de Early Upper Paleolithic, ont livré des lamelles retouchées et des pointes à retouche inverse de type el-Wad. Cette composante lamellaire en faible quantité de Tor Sadaf B et A coexiste avec des pointes Levallois (Fox 2003). Dans le niveau sus-jacent au Early Upper Paleolithic, les pointes Levallois diminuent tandis que les pointes de el-Wad et les lamelles retouchées constituent la moitié de l'outillage (Fox and Coinman 2004). La transition Paléolithique moyen/Paléolithique supérieur dans le site de Tor-Sadaf $A$ et $B$ et le Paléolithique supérieur ancien ont un âge estimé entre 43 - 38000 BP (Fox 2003).

En Europe du sud-ouest, nous trouvons des industries lamellaires du début du Paléolithique supérieur redéfinies récemment comme Proto-Aurignacien (ou Aurignacien archaïque) (Bon 2002). Le Proto-Aurignacien méditerranéen qui est postérieur au Châtelperronien et précède l'Aurignacien ancien (à grattoirs carénés) est généralement daté entre 38- 31 Ky (Bon 2002 ; Teyssandier 2003). Dans la grotte Fumane (Broglio et al. 1996) ce faciès se caractérise par de grandes lamelles rectilignes (environ $40 \mathrm{~mm}$ ), provenant de nucléus prismatiques ou pyramidaux et de plus petites lamelles $(25 \mathrm{~mm})$ souvent courbes, issues de nucléus "carénoïdes ". Les sites de Riparo Mochi (couche G) (Kuhn and Stiner 1998), Arbreda (couche H) (Maroto et al. 1996 ; Ortega Cobos et al. 2005), Arcy-sur-Cure (couche VII) (Bon et Bodu 2002), et le Piage (couche K) (Bordes 2002) ont livré des industries à grandes lamelles retouchées, issues d'un débitage unipolaire semi-toumant identique à celui des nucléus à lames.

\section{RÉFLEXION SUR LA PRÉSENCE DES LAMELLES À DOS DANS LES PHASES INITIALES DE L'AURIGNACIEN}

Nous avons discuté la présence de quelques lamelles à dos épais dans le cadre d'une bonne série de pièces à dos minces dans l'ensemble du niveau VII de Kozarnika. On a pu constater, pour les sites de comparaisons, que dans le cadre des aspects communs les plus généraux (production et outillage lamellaire) c'est la présence de pièces à dos qui reste négligée.

Par exemple, la couche VII de la grotte du Renne (à Arcy-sur-Cure) a livré une industrie lithique riche en grandes lamelles Dufour rectilignes (plus de 380 exemplaires) et une vingtaine de lamelles à dos (Perpère et Schmider 2002). Les dimensions, la retouche (irrégulière) et la latéralisation (aléatoire) de ces dernières ne les rapprochent pas des lamelles Dufour. Les auteurs ne les décrivent pas précisément.

A Riparo Mochi, les lamelles à dos sont beaucoup plus importantes car ce type d'outils représente en effet presque $6 \%$ de l'outillage, bien que les lamelles Dufour constituent plus de $30 \%$ (Kuhn and Stiner 1998). En tout les lamelles retouchées et à dos représentent plus de $40 \%$ de l'outillage.

La présence négligée des lamelles à dos semble peut-être ne pas avoir une grande importance mais à cela, s'ajoutent d'autres négligences ou surestimations qui déforment nos conclusions. La reconnaissance de telles déformations commence déjà à provoquer de nouvelles propositions dans le débat sur la transition Paléolithique moyen/Paléolithique supérieur et la formation des premières unités culturelles du Paléolithique supérieur. Le cas de Kozarnikien nous montre d'une part, que la formation des industries à pièces à dos a commencé bien avant 30 $\mathrm{Ky}$, probablement contemporaine des phases précoces des autres traditions et, d'autre part, que certaines de ces industries lamellaires initiales pouvaient avoir des racines très proches sinon communes à celles de la partie est de la zone méditerranéenne ${ }^{3}$. En ce qui concerne l'Europe, d'après l'état actuel de nos connaissances, les premières traces de ces industries sont concentrées dans la partie sud du continent.

En même temps, la présence de lamelles à dos dans plusieurs ensembles aurignaciens et leur absence dans les autres s'ajoutent aux arguments qui ne confirment pas l'uniformité de l'Aurignacien. II nous semble aussi qu'on ne puisse plus soutenir la conception du rôle exclusif de l'Aurignacien dans la transition de toute l'Europe moustérienne vers le Paléolithique supérieur. II devient de plus en plus évident qu'au tout début de cette transformation, des traditions culturelles lamellaires différentes de l'Aurignacien ont été engagées.

\section{CONCLUSION}

La position chronostratigraphique et les caractères techno-typologiques du Kozarnikien ancien (niveau VII) suggèrent un rapprochement préliminaire et plus général avec les faciès originaux lamellaires du début du Paléolithique supérieur représentés comme nous le voyons dans les régions du Proche-Orient et de l'Europe méditerranéenne. En même temps, en l'absence des éléments caractéristiques de l'Aurignacien typique, cette industrie montre des tendances dont on trouve la continuité dans les niveaux sus-jacents gravettiens de Kozarnika, et, de ce point de vue, elle peut représenter une étape précoce de formation du technocomplexe lamellaire à pièces à dos.

L'état actuel des recherches sur le Paléolithique bulgare et la faible quantité de sites de références stratifiés (seule-

(3) II nous semble indispensable de rappeler ici l'hypothèse de synthétotype aurignaco-Périgordien proposé par G. Laplace. Nous renvoyons le lecteur à deux articles importants à ce titre (LAPLACE G. 1956 - Typologie statistique et évolution des complexes à lames et lamelles. Bulletin de la Société Préhistorique Française, p. 271-290 et LAPLACE G. 1970 - Les niveaux aurignaciens et l'hypothèse du synthétotype. In : L'Homme de Cro-Magnon. Centre de Recherche anthropologique, préhistorique et ethnographique, Paris, p. 141-164). 
ment trois pour le début de Paléolithique supérieur) limitent nos connaissances sur la variabilité de cette tradition technologique. Cependant, des comparaisons plus poussées de la variabilité régionale de ce faciès restent à effectuer en cherchant de nouveaux sites aux alentours dans la région ou sur la voie danubienne.

La révision récente des faciès lamellaires les plus anciens en Europe centrale (Teyssandier 2003) a montré l'absence d'une phase stratifiée antérieure de l'Aurignacien ancien, ce qui suggère une même problématique de diffusion de cette culture sur la voie danubienne. Y-a-t-il une limite de diffusion de cette tradition technologique lamellaire du début de Paléolithique supérieur ou est-ce l'insuffisance de l'état actuel de la recherche ? Nous n'avons pas de traces de ces premières industries lamellaires en Europe centrale, bien que les complexes de transition soient représentés du Proche Orient (Boker Tachtit) aux Balkans (le Bachokirien et vraisemblablement Temnata couche VI) jusqu'à l'Europe centrale (le Bohunicien).

\section{Remerciements}

Les fouilles à Kozarnika sont réalisées dans le cadre d'un projet conjoint " Les plus anciennes manifestations de la présence humaine dans les Balkans" (directeurs N. Sirakov et J.-L. Guadelli) entre I'Institut National d'Archéologie de l'Académie bulgare des sciences et l'Institut de Préhistoire et de Géologie du Quatemaire, PACEA-UMR 5199 du CNRS (France). Les opérations de terrain et les analyses sont soutenues financièrement par la Commission Consultative des Recherches Archéologiques au Ministère des Affaires Etrangères (DRI, UMR 5199), la Région Aquitaine (France), l'Académie bulgare des Sciences et par le Max-Planck Institut de Leipzig (Allemagne), à qui nous adressons nos plus vifs remerciements.

Nous remercions également $\mathrm{V}$. Laroulandie et $\mathrm{M}$. Lenoir pour la relecture de cet article et pour les discussions.

\section{BIBLIOGRAPHIE}

BEELFER-COHEN A. and N. GORING-MORRIS

2003 - Current Issues in Levantine Upper Palaeolithic Research In More Than Meets The Eye. Studies on Upper Palaeolithic Diversity in the Near East, edited by A. N. Goring-Morris and A. Belfer-Cohen, pp. 1-12. Oxford, Oxford.

BON F. 2000 - La question de l'unité technique et économique de l'Aurignacien : Réflexions sur la variabilité des industries lithiques à partir de l'étude comparée de trois sites des Pyrénées françaises (La Tuto de Camalhot, Régismont-le-Haut et Brassempouy), Thèse de doctorat de l'Université Paris I - Panthéon - Sorbonne.

BON F. 2002 - L'Aurignacien entre mer et Océan. Réflexion sur l'unité des phases anciennes de l'Aurignacien dans le Sud de la France t XXIX, Paris.
BON F. et BODU P. 2002 - Analyse technologique du débitage aurignacien. In L'Aurignacien de la grotte du Renne. Les fouilles d'André Leroi-Gourtan à Arcy-sur-Cure (Yonne), éd. B. Schmider, pp. 115-141. vol. XXXIVe supplément à Gallia Préhistoire. CNRS édition, Paris.

BORDES J.-G. 2002 - Les interstratifications Châtelperronien / Aurignacien du Roc-de-Combe et du Piage (Lot, France). Analyse taphonomique des industries lithiques ; implications archéologiques., Thèse de doctorat de l'Université Bordeaux I.

BROGLIO A., ANGELUCCI D. E., PERESANI M., LEMORINI C. and ROSSETTI P. 1996 - L'industrie protoaurigna cienne de la grotta di Fumane : données préliminaires. Actes du XIII congrès de I'UISPP, Forli, Italie, septembre 1996, vol.2 : 495-509.

DEMARS P.-Y. et LAURENT P. 1992 - Types d'outils lithiques au Paléolithique supérieur en Europe. CNRS éd., Paris.

FERRIER C. et LEBLANC J.-C. 2004 - Géologie, cartogra phie, géomorphologie, karstologie. Description stratigraphique des dépôts de Kozarnika. In Guadelli J.-L. \& Sirakov N. éds. : Les plus anciennes manifestations de la présence humaine en Bulgarie du nord «, Rapport de $P$ rojet de recherche conjoint, exemplaire remis au Ministère des Affaires Etrangères de France, pp. 20-56.

FOX J. R. 2003 - The Tor Sadaf Lithic Assemblages : A Technological Study of the Early Upper Palaeolithic in the Wadi al-Hasa. In More Than Meets The Eve. Studies on Upper Palaeolithic Diversity in the Near East., edited by A. N. Goring-Morris and A. Belfer-Cohen, pp. 80-94. Oxbow Books, Oxford.

FOX J. R. and COINMAN N. R. 2004 - Emergence of the Levantine Upper Paleolithic. Evidence from the Wadi alHasa. In The Early Upper Paleolithic beyond Westem Europe, edited by P. J. Brantingham, S. L. Kuhn and K.W. Kerry, pp. 97-112. University of California Press, Berkeley Los Angeles London.

GORING-MORRIS A. N. and BELFER-COHEN A. (editors) 2003 - More Than Meets The Eve. Studies on Upper Palaeolithic Diversity in the Near East. Oxbow Books, Oxford.

GUADELLI J.-L. et SIRAKOV N. 2004 (éditeurs) - Les plus anciennes manifestations de la présence humaine en Bulgarie du nord, Rapport de Projet de recherche conjoint, exemplaire remis au Ministère des Affaires Etrangères de France).

GUADELLI J.-L., SIRAKOV N. et IVANOVA S., SIRAKOVA S., ANASTASSOVA E., COURTAUD P., DIMITROVA I., DJABARSKA N., FERNANDEZ, Ph. FERRIER C., FONTUGNE M., GAMBIER D., GUADELLI A., IORDANOVA D., IORDANOVA N., KOVATCHEVA M., KRUMOV I., LEBLANC J.-Cl., MALLYE J.-B., MARINSKA M., MITEVA 
V., POPOV V., SPASSOV R., TANEVA St., TISNERATLABORDE N., TSANOVA, Ts. 2005 - Une séquence du Paléolithique inférieur au Paléolithique récent dans les Balkans: la grotte Kozarnika à Orechets (Nord-Ouest de la Bulgarie). In: Colloque intérnational: Données récentes sur les modalités de peuplement et sur le cadre chronostrati graphique des industries du Paléolitique inférieur et moyen en Europe., Renne, 2003, 22-25 septembre, edited by N. Molines, M.-H. Moncel and J.-L. Monnier. vol. S 1364. British Archeological Reports.

KUHN S. L. and STINER M. C. 1998 - The Earliest Aurignacian of Riparo Mochi (Liguria, Italy). Current Anthropology 39(3):175-189.

LENOIR M. - 2001 Les niveaux supérieurs gravettiens de la grotte Kozarnika (niveaux I, II, II-III, IVa). In : Guadelli J.L. \& Sirakov N. éds. : Les plus anciennes manifestations de la présence humaine en Bulgarie du nord ", Rapport de $P$ rojet de recherche conjoint, exemplaire remis au Ministère des Affaires Etrangères de France, pp. 181-186.

LUCAS G. 2000 - Le niveau VI inférieur de la grotte Kozarnika (Bulgarie) : quelle identification culturelle? In : Guadelli J.-L. \& Sirakov N. éds. : Les plus anciennes manifestations de la présence humaine en Bulgarie du nord ", Rapport de Projet de recherche conjoint, exemplaire remis au Ministère des Affaires Etrangères de France, pp.151-152.

MAROTO J., SOLER N. and. FULLOLA J. M 1996 Cultural change between Middle and Upper Palaeolithic in Catalonia In : The Last Neandertals, The first anatomically Modem Humans : A Tale About The Human Diversity. Cultural change and human evolution : the crisis at $40 \mathrm{Ka}$ $B P$, edited by E. Carbonell and M. Vaquero, pp. 219-250, Barcelona.

MONIGAL K. 2003 - Technology, Economy and Mobility at the Beginning of the Levantine Upper Palaeolithic In More Than Meets The Eye. Studies on Upper Palaeolithic Diversity in the Near East, edited by A. N. Goring-Morris and A. Belfer-Cohen, pp. 118-133. Oxbow Books, Oxford.

NATCHEV C. 2003 - Les niveaux de silex prés de la grotte Kozamika dans l'optique de leur utilisation par les préhistoriques In Guadelli J.-L. \& Sirakov N. éds. : Les plus anciennes manifestations de la présence humaine en Bulgarie du nord «, Rapport de Projet de recherche conjoint, exemplaire remis au Ministère des Affaires Etrangères de France, pp. 30-34.

ORTEGA COBOS D., SOLER MASFERRER N. and MAROTO GENOVER J. 2005 - La production des lamelles pendant l'Aurignacien archaïque dans la grotte de l'Arbreda : organisation de la production, variabilité des méthodes et des objectifs. In Productions lamellaires attribuées à l'Aurignacien : Chaînes opératoires et perspectives technoculturelles XIVe congrès de I'UISPP, Liège 2-8 Septembre 2001, edited by F. Le Brun-Ricalens, J.-G. Bordes and F. Bon, pp. 359-373. vol. ArchéoLogique 1.
Imprimerie Fr. Faber, Mersch, Grand-Duché de Luxembourg, Luxembourg.

OTTE M. et KOZLOWSKI J. K. 2004 - La place du Baradostien dans l'origine du Paléolithique supérieur d'Eurasie. l'Anthropologie 79:1-12.

PELEGRIN J. 2000 - Les techniques de débitage laminaire au Tardiglaciaire : critères de diagnose et quelques réflexions In L'Eurqpe centrale et septentrionale au Tardiglaciaire Eds. B. Valentin, P. Bodu et M. Christensen, (Mémoires du Musée de Préhistoire d'lle de France, 7), Actes de la table-ronde de Nemours : 73-86.

PERPERE M. et SCHMIDER B. 2002 - L'outillage lithique. In L'Aurignacien de la grotte du Renne. Les fouilles d'André Leroi-Gourhan à Arcy-sur-Cure (Yonne), Ed. B. Schmider, pp. 143-192. vol. XXXIVe supplément à Gallia Préhistoire. CNRS édition, Paris.

POPOV R. 1933 - Pechterata Mirizlivka. Prinos kum diluvialnata fauna i kulturata na diluvialnia tchovek v Bulgaria. (La grotte Mirizlivka. Contribution à l'étude de la faune diluvienne et de la culture de l'homme diluvien en Bulgarie.). Izvestia na natsionalnia arheologitcheski museî, Izvestia na natsionalniat archeologitcheski museî, Cahier du musée national d'Archéologie, Sofia 26:5-69.

RIGAUD J.-Ph. 2001 - A propos de la contemporanéité du Castelperronien et de l'Aurignacien ancien dans le nord-est de l'Aquitaine : une révision des données et ses implication In : Les premiers hommes modernes de la Péninsule Ibérique. Actes du Colloque de la Commission VIII de I'UISPP, Vila Nova de Foz Côa, 22-24 Octobre 1988, J. Zilhao, T. Aubry, AF Carvalho, eds. Instituto Português de Arqueologia, Lisboa p. 61-67.

RIGAUD J.-Ph. et LUCAS G. 2006 - The first aurignacian techno-complex in Europe : a revision of the Bachokirian. In: "Toward a definition of the Aurignacian ". Proceeding of the Symposium held inLisbon, Portugal, June 25-30, 2002 ; O. Bar Yosef \& J. Zilhao eds. Instituto Portuguès de Archeologia, Lisboa, 2006, p. 277-284.

SONNEVILLE-BORDES D.(de) et PERROT J.1954 Lexique typologique du Paléolithique supérieur. Outillage lithique - I grattoirs, II outils solutréens. Bulletin de la Société Préhistorique Française 51(7):327.

TEYSSANDIER N. 2003 - Les débuts de l'Aurignacien en Europe. Discussion à partir des sites de Geissenkösterle, Wilendorf II, Krems-Hundssteig et Bacho Kiro, Thèse de doctorat de l'Université de Paris X-Nanterre.

TSANOVA T. 2001 - Etude techno-typologique du niveau gravettien IVb de la grotte Kozarnika (Bulgarie du Nord), Mémoire de DEA de l'Université de Bordeaux I.

TSANOVA T. 2003 - Le Gravettien en Bulgarie du Nord : niveau IVb de la grotte Kozamika In: The Humanized Mineral Word: Towards social and symbolic evaluation of 
prehistoric technology in South Eastern Europe, Eds. T. Tsonev \& E.M. Kokelj, Proceedings of the ESF workshop, Sofia, 3-6 september, 2003 : 33-40.

TSANOVA Ts. 2006 - Les débuts du Paléolithique supérieur dans l'Est des Balkans. Réflexion à partir de l'étude taphonomique et techno-économique des ensembles lithiques de Bacho Kiro (couche 11), Temnata (couches VI et 4) et Kozarnika (niveau VII), Thèse de doctorat, Université de Bordeaux 1.
TSANOVA Ts. et BORDES J.-G. 2003 - Contribution au débat sur l'origine de l'Aurignacien : principaux résultats d'une étude technologique de l'industrie lithique de la couche 11 de Bacho Kiro. In: The Humanized Mineral Word: Towards social and symbolic evaluation of prehistoric technology in South Eastern Europe, Eds. T. Tsonev \& E.M. Kokelj, Proceedings of the ESF workshop, Sofia, 3-6 september, $2003:$ 41-50. 